\title{
Serum-Soluble CD40 Ligand Level in MPO-ANCA-Associated Renal Vasculitis
}

Kouichi Hirayama $^{1 *}$, Miho Nagai ${ }^{2}$, Itaru Ebihara ${ }^{3}$, Takashi Higuchi ${ }^{4}$, Hiroshi Maruyama ${ }^{1}$, Yasunori Miyamoto ${ }^{1}$, Yujiro Ogawa ${ }^{1}$, Homare Shimohata ${ }^{1}$ and Masaki Kobayashi

${ }^{1}$ Department of Nephrology, Tokyo Medical University Ibaraki Medical Center, Ami, Ibaraki, Japan

${ }^{2}$ Department of Nephrology, Tokyo Medical University, Shinjuku-ku, Tokyo, Japan

${ }^{3}$ Department of Nephrology, Mito Saiseikai General Hospital, Mito, Ibaraki, Japan

${ }^{4}$ Department of Immunology, Faculty of Medicine, University of Tsukuba, Tsukuba, Ibaraki, Japan

Corresponding author: Kouichi Hirayama, Department of Nephrology, Tokyo Medical University Ibaraki Medical Center, 3-20-1 Chuo, Ami, Ibaraki, 300-0395 Japan, Tel.: +81 29 8871161; E-mail: k-hira@tokyo-med.ac.jp

Received date: September 20, 2015; Accepted date: November 25, 2015; Published date: December 2, 2015

Copyright: @ 2015 Hirayama K et al. This is an open-access article distributed under the terms of the Creative Commons Attribution License, which permits unrestricted use, distribution, and reproduction in any medium, provided the original author and source are credited.

\begin{abstract}
Purpose: The CD40/CD40 ligand (CD40L) system is associated with pathogenic processes of inflammatory diseases, and elevated levels of soluble CD40L have been observed in sera from patients with autoimmune diseases. To determine the diagnostic value of serum-soluble CD40L in Antineutrophil cytoplasmic antibody (ANCA)associated vasculitis (AAV), we investigated patients with MPO-AAV at various stages of the disease.

Methods: Twenty-eight samples were from patients with active MPO-AAV: 28 samples from inactive-vasculitis patients without infection and 16 samples from inactive-vasculitis patients with infectious complications. Serum soluble CD40L was measured by an enzyme-linked immunosorbent assay. Serum-soluble CD40L was also measured in 15 patients with infectious diseases and in 28 control subjects.

Results: The serum-soluble CD40L level was higher in the patients with inactive vasculitis with infectious complications than in those with inactive vasculitis without infection and the normal control, but there was no significant difference in CD40L levels between the patients with active vasculitis and those with inactive vasculitis with infectious complications. There were significant correlations between the serum levels of soluble CD40L and the serum creatinine and CRP levels. The serum soluble CD40L/creatinine ratio was higher in the inactive vasculitis with infectious complications group than in the active vasculitis group, inactive vasculitis without infection group and the normal control. Comparative receiver-operating-characteristic curves showed that this ratio had $92.8 \%$ sensitivity and $96.8 \%$ specificity for differentiating patients with infection from those without infection.
\end{abstract}

Conclusion: Serum-soluble CD40L was not associated with disease activity, but it may be a useful marker for the detection of infectious complications in MPO-AAV.

Keywords: ANCA-associated vasculitis; Microscopic polyangiitis; CD40 ligand; Disease activity; Infectious complications

\section{Introduction}

In antineutrophil cytoplasmic antibody (ANCA)-associated vasculitis (AAV), the diagnostic value of assays for ANCAs is now widely accepted. ANCAs are known to bind two key antigens found in neutrophil granules and monocyte lysosomes: proteinase-3 (PR3) and myeloperoxidase (MPO). ANCAs can activate cytokine-primed neutrophils, causing an oxidative burst, degranulation, the release of inflammatory cytokines, and damage to endothelial cells in vitro [1], and an intravenous injection of mouse antibodies specific for mouse MPO induced pauci-immune necrotizing and crescentic glomerulonephritis in a mouse model that closely mimics the human disease [2]. The pathogenicity of anti-MPO antibodies has been demonstrated in vitro and in vivo [1-3], but the mechanisms of MPOANCA production have not been clarified. Moreover, the MPO-ANCA titer is not always associated with disease activity.

The interaction between CD40 and its ligand (CD40L) is critical to the control of thymus-dependent humoral immunity and cell- mediated immune responses [4]. CD40L on the $\mathrm{T}$ cells stimulates the B-cell secretion of immunoglobulin isotypes in the presence of cytokines. The interaction of CD40L with CD40 not only induces a proliferation of $\mathrm{B}$ lymphocytes and their isotype switching; it also mediates a broad variety of other immune and inflammatory responses [4]. CD40 signaling has been linked with pathogenic processes of chronic inflammatory diseases such as autoimmune diseases, neurodegenerative disorders and graft-versus-host disease [5]. Soluble forms of CD40L, produced by proteolytic cleavage, lack the transmembrane region and a portion of the extracellular domain, but they contain the entire tumor necrosis factor-alpha (TNF- $\alpha$ ) homology region [6]. Elevated levels of soluble CD40L have been observed in sera from patients with systemic lupus erythematosus (SLE) [7,8], rheumatoid arthritis with vasculitis [9], Sjögren's syndrome [8], mixed connective tissue disease [10], systemic sclerosis [11] and Kawasaki disease [12], and a direct relationship can be seen between serumsoluble CD40L and the disease severities or titers of autoantibodies (anti-double-strand DNA antibody) [7] and rheumatoid factor [8]. In patients with granulomatosis with polyangiitis (GPA), soluble CD40L was associated with disease activity [13]. 
We hypothesized that CD40L may be released from $\mathrm{T}$ cells or damaged tissue in AAV, and in the present study we investigated patients with micropolyangiitis (MPA) at various stages of the disease to determine the diagnostic value of serum-soluble CD40L in MPOAAV.

\section{Material and Methods}

\section{Subjects}

The cases of 43 Japanese MPO-ANCA associated MPA patients were examined. The diagnosis of MPA was based on the European Medicines Agency algorithm [14]. Patients with GPA or Eosinophilic granulomatosis with polyangiitis (EGPA) were excluded. ANCA was examined using an MPO-specific Enzyme-linked immunosorbent assay (ELISA), and normal ranges were defined as values below 3.5 $\mathrm{U} / \mathrm{ml}$. Patients with a positive test result for proteinase-3-ANCA and anti-glomerular basement membrane antibody were excluded.

Among the 43 MPO-AAV patients, 28 provided serum samples before the initial treatment, and 28 provided samples during remission (15 provided samples both before the initial treatment and during remission). Remission was defined as the absence of clinical disease activity based on the Birmingham vasculitis activity score (BVAS). Sixteen samples obtained during remission with infectious complications were investigated. Ten of the 16 patients with infectious complications had bacterial pneumonia, four had acute bronchitis, one had pulmonary tuberculosis, and the remaining patients had bacterial pleuritis.

As positive infectious control, 10 patients with acute pyelonephritis and five patients with bacterial pneumonia were investigated. The diagnosis of acute pyelonephritis was based on characteristic clinical features such as fever, costovertebral tenderness, leukocyturia, bacteria in urine sediment and urinary culture examination, elevated White blood cell (WBC) count and elevated serum C-reactive protein (CRP) level. The normal range of serum CRP is defined as a value below 0.3 $\mathrm{mg} / \mathrm{dL}$. The diagnosis of bacterial pneumonia was based on characteristic clinical features such as fever, cough, sputum, elevated WBC count, serum CRP level, and chest X-ray findings.

As normal control, eight healthy volunteers, 15 patients with Chronic kidney disease (CKD) and five acute pyelonephritis patients after antibiotic treatment were investigated. Three of the 15 patients with CKD had nephrosclerosis, 10 patients had chronic glomerulonephritis (including one patient treated with hemodialysis), and two patients had diabetic nephropathy. Patients with elevated levels of serum CRP were excluded from the group of normal control.

The study protocol was accepted by the Ethics Committee of Tokyo Medical University Ibraki Medical Center, and written informed consent was obtained from all patients or their immediate family members. This study also conformed to the provisions of the Declaration of Helsinki as revised in Edinburgh in 2000.

\section{ELISA for soluble CD40L}

Blood samples were collected in plasma separator tubes before initial treatment, during remission status, and at the time of complicating infection. Samples were separated at $1000 \times \mathrm{g}$ for $15 \mathrm{~min}$ and were stored at $-80^{\circ} \mathrm{C}$ for analysis.

The samples were measured by commercially available ELISA kits (Quantikine ${ }^{\oplus}, \mathrm{R} \& D$ Systems, Minneapolis, MN) in duplicate. In brief, 98-well microplates were first coated with polyclonal anti-human CD40L antibody. Then, $100 \mu \mathrm{l}$ of fivefold-diluted sample was added to each well. Plates were incubated for $2 \mathrm{~h}$ at room temperature (RT) and then washed, and horseradish peroxidase-conjugated polyclonal antihuman CD40L antibody was added at $200 \mu \mathrm{l} /$ well. The plates were incubated for $2 \mathrm{~h}$ at RT and then washed, and $100 \mu \mathrm{l}$ of chromogen (tetramethylbenzidine) and $100 \mu \mathrm{l}$ of hydrogen peroxide were added to each well. The plates were incubated for $30 \mathrm{~min}$ at RT, and $50 \mu \mathrm{l}$ of $2-\mathrm{N}$ sulfuric acid solution was added to each well. The plates were immediately read on a microplate reader (Sunrise Remote, Tecan Japan, Kanagawa, Japan) set at $450 \mathrm{~nm}$ for measurement and at 540 $\mathrm{nm}$ for wavelength correction. The inter and intra-assay variations were less than $10 \%$.

\section{Statistical analysis}

Continuous variables are presented as mean \pm Standard deviation (SD), and categorical variables are presented as numbers with percentages. Differences in categorical variables were checked using the chi-square test with Yates' continuity correction and Fisher's exact test, and post-hoc comparisons (Bonferroni correction) were performed to detect differences among three or more groups. An analysis of variance (ANOVA) was used to assess differences among subject groups, and post-hoc comparisons were made using the Bonferroni/Dunn test. A correlation analysis was performed using Spearman's univariate correlation coeffcient ( $r$ ) to determine correlations between the serum soluble CD40L levels and clinical parameters such as age, BVAS, MPO-ANCA titer, WBC count, hemoglobin concentration, platelet count, and serum creatinine and CRP levels.

We performed a multiple linear regression analysis that included the covariates found to be significantly associated with the serum soluble $\mathrm{CD} 40 \mathrm{~L}$ level in the correlation analysis. The data are expressed as standardized regression coefficients $(\beta)$. P-values $<0.05$ were accepted as significant, but in the multiple comparisons, the critical P-value was divided by the number of comparisons being made. The comparative Receiver operating characteristic (ROC) curves for inflammatory variables of disease activity and the diagnosis of infection were obtained. All statistical analyses were performed using PASW Statistics software, version 18 (IBM Japan [formerly SPSS Japan Inc.], Tokyo) for Windows.

\section{Results}

\section{Clinical parameters of subjects}

There was no significant difference in age among the patients of the five groups: (1) active MPO-AAV patients, (2) inactive-vasculitis patients without infection, (3) inactive-vasculitis patients with infectious complications, (4) infectious control, and (5) normal control, but the percentage of males was significantly lower in the patients with infectious control (Table 1). The MPO-ANCA titer was significantly higher in the patients with active vasculitis than in the other four groups, and MPO-ANCA was not detected in either control group. Leukocytosis and elevated serum CRP levels were observed in the patients with active vasculitis and the infectious groups (inactive vasculitis with infectious complications and the infectious control). Elevated serum creatinine levels were observed in the active-vasculitis patients. 


\begin{tabular}{|c|c|c|c|c|c|c|}
\hline & Units & $\begin{array}{l}\text { Active } \\
(\mathrm{n}=28)\end{array}$ & $\begin{array}{l}\text { Inactive } \quad \text { vasculitis } \\
(n=28)\end{array}$ & $\begin{array}{l}\text { Inactive vasculitis with } \\
\text { infections }(n=16)\end{array}$ & $\begin{array}{l}\text { Infectious } \\
(n=15)\end{array}$ & $\begin{array}{l}\text { Normal control } \\
(n=28)\end{array}$ \\
\hline Age & (yrs) & $69.6 \pm 14.14$ & 70.7 & $70.4 \pm 9.8$ & $70.3 \pm 18.4$ & $68.8 \pm 15.4$ \\
\hline Gender (male) & - & $17(61 \%)$ & $14(50 \%)$ & $9(56 \%)$ & $2(13 \%)^{a, c, d, e}$ & $13(46 \%)$ \\
\hline BVAS & - & $20.7 \pm 5.2$ & $0 \pm 0$ & $0 \pm 0$ & - & - \\
\hline MPO-ANCA & $(\mathrm{U} / \mathrm{ml})$ & $307.4 \pm 170.3^{a, b, c, c, d}$ & $21.4 \pm 38.3$ & $51.3 \pm 115.9$ & $1.3 \pm 0$ & $1.3 \pm 0$ \\
\hline White blood cell & $\left(/ \mathrm{mm}^{3}\right)$ & $9704 \pm 3879$ a & $8414 \pm 2342$ & $12131 \pm 3090^{a, d}$ & $13080 \pm 5156^{\mathrm{a}, \mathrm{d}, \mathrm{e}}$ & $6122 \pm 1502$ \\
\hline Hemoglobin & $(\mathrm{g} / \mathrm{dl})$ & $9.9 \pm 2.2^{\mathrm{a}, \mathrm{b}}$ & $11.5 \pm 1.9$ & $10.8 \pm 2.8$ & $12.4 \pm 1.8$ & $11.8 \pm 2$ \\
\hline Platelet & $\left(\times 10^{4} / \mathrm{mm}^{3}\right)$ & $27.1 \pm 13.2$ & $22.1 \pm 4.7$ & $20.1 \pm 10.1$ & $19.9 \pm 7.3$ & $22.6 \pm 6.1$ \\
\hline Serum albumin & $(g / d)$ & $3.04 \pm 0.61 \mathrm{a}, \mathrm{b}, \mathrm{d}$ & $3.76 \pm 0.38$ & $3.27 \pm 0.78^{a, d}$ & $3.75 \pm 0.44$ & $4.05 \pm 0.36$ \\
\hline Serum creatinine & $(\mathrm{mg} / \mathrm{dl})$ & $4.41 \pm 2.59 \mathrm{a}, \mathrm{b}$ & $2.92 \pm 3.34$ & $2.89 \pm 2.24$ & $1.26 \pm 0.73$ & $1.76 \pm 1.51$ \\
\hline Serum CRP & $(\mathrm{mg} / \mathrm{dl})$ & $7.15 \pm 6.56 \mathrm{a}, \mathrm{d}$ & $0.22 \pm 0.14$ & $8.75 \pm 5.42^{\mathrm{a}, \mathrm{d}}$ & $12.43 \pm 7.46^{\mathrm{a}, \mathrm{d}, \mathrm{e}}$ & $0.11 \pm 0.15$ \\
\hline \multicolumn{7}{|c|}{$\begin{array}{l}\text { Variables are expressed as means } \pm S D \text { or as numbers with percentages of the total. } \\
\text { aP }<0.005 \text { vs. Normal control; }{ }^{b} P<0.005 \text { vs. Infectious control; }{ }^{\circ}<<0.005 \text { vs. Inactive-vasculitis with infection; }{ }^{d} P<0.005 \text { vs. Inactive-vasculitis; }{ }^{e} P<0.005 \text { vs. Active- } \\
\text { vasculitis. }\end{array}$} \\
\hline \multicolumn{7}{|c|}{ BVAS: Birmingham Vasculitis Activity Scores; } \\
\hline \multicolumn{7}{|c|}{ MPO: Myeloperoxidase; } \\
\hline \multicolumn{7}{|c|}{ ANCA: Antineutrophil Cytoplasmic Antibody; } \\
\hline \multicolumn{7}{|c|}{ CRP: C-Reactive Protein. } \\
\hline
\end{tabular}

Table 1: Characteristics of the five groups of subjects.

\section{Serum levels of soluble CD40L}

Among the patients with MPO-AAV, the serum levels of soluble CD40L were significantly higher in the inactive-vasculitis patients with infectious complications compared to the inactive-vasculitis patients and normal control (Figure 1). However, there was no significant difference in CD40L levels between the active vasculitis patients and those with inactive vasculitis with infectious complications.

There was no significant correlation between the serum levels of soluble CD40L and age, BVAS, or MPO-ANCA titers (Table 2). There were significant positive correlations between the serum levels of serum-soluble CD40L and the serum creatinine levels, WBC count, hemoglobin concentration, and serum CRP levels. In the multiple regression analysis including the above four variables, there were significant positive correlations between the serum levels of serumsoluble CD40L and the serum creatinine levels, WBC count and serum CRP levels.

\section{Ratio of serum-soluble CD40L/creatinine}

To remove the factor of renal function, we calculated the ratio of serum-soluble CD40L to creatinine. Among the patients with MPO$\mathrm{AAV}$, the serum ratio of soluble CD40L to creatinine was higher in the inactive-vasculitis patients with infectious complications than in those with active vasculitis or inactive vasculitis without infection, and the normal control (Figure 2). That ratio was also higher in the infectious control than in the patients with inactive vasculitis without infection and the normal control.

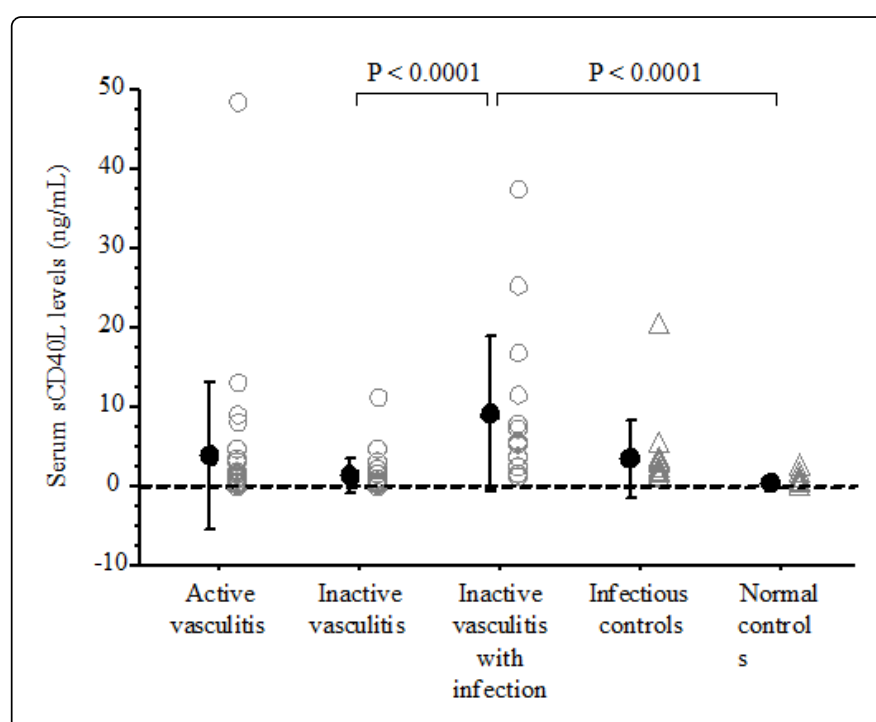

Figure 1: Serum levels of soluble CD40 ligand in the five subject groups. Black closed circles and bars show means and standard errors. Gray open circles=MPO-ANCA-associated vasculitis. Gray open triangles=individual values of the control.

\begin{tabular}{|c|c|c|c|c|}
\hline \multirow[b]{2}{*}{ Clinical Parameters } & \multicolumn{2}{|c|}{ Univariate } & \multicolumn{2}{|c|}{ Multivariate } \\
\hline & $\mathbf{r}$ & P-value & $\beta$ & P-value \\
\hline Age & 0.035 & 0.7121 & - & - \\
\hline
\end{tabular}


Citation: Hirayama K, Nagai M , Ebihara I, Higuchi T, Maruyama H, et al. (2015) Serum-Soluble CD40 Ligand Level in MPO-ANCA-Associated Renal Vasculitis. J Vasc 1: 102. doi:10.4172/2471-9544.1000102

Page 4 of 7

\begin{tabular}{|l|l|l|l|l|}
\hline BVAS & 0.067 & 0.5789 & - & - \\
\hline MPO-ANCA & 0.082 & 0.3843 & - & - \\
\hline White blood cell & 0.445 & $<0.0001$ & 0.29 & 0.0108 \\
\hline Hemoglobin & -0.229 & 0.0141 & -0.100 & 0.2789 \\
\hline Platelet & 0.003 & 0.9747 & - & - \\
\hline Serum creatinine & 0.26 & 0.005 & 0.213 & 0.0211 \\
\hline Serum CRP & 0.441 & $<0.0001$ & 0.225 & 0.0488 \\
\hline
\end{tabular}

BVAS: Birmingham Vasculitis Activity Scores; MPO: Myeloperoxidase; ANCA Antineutrophil Cytoplasmic Antibody; CRP: C-Reactive Protein

Table 2: Correlation and regression coefficiencies between clinical parameters and serum soluble CD40L levels in MPO-ANCAassociated vasculitis.

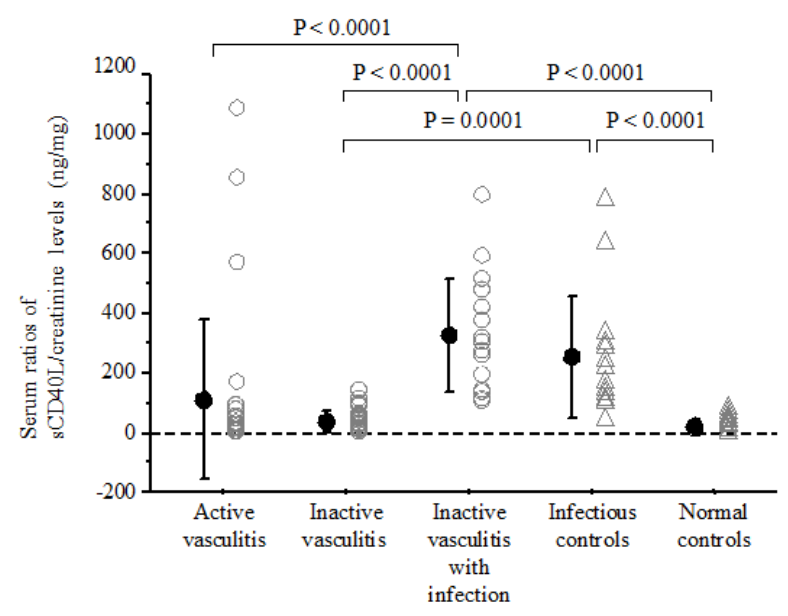

Figure 2: Serum ratios of soluble CD40L to creatinine in the five subject groups. Black closed circles and bars=means and std. errors. Gray open circles=MPO-ANCA associated vasculitis. Gray open triangles=individual values of the control.

\section{ROC curve analysis}

The comparative ROC curves for three measures of disease activity (WBC counts, serum CRP levels, and the ratio of serum-soluble CD40L to creatinine) and the diagnosis of infection are shown in Figure 3. The optimum cut-off levels for the diagnosis of infection were identified from the ROC curves for WBC counts, serum CRP levels and the serum ratio of $\mathrm{CD} 40 \mathrm{~L}$ to creatinine. The area under the curve of the serum ratio of $\mathrm{CD} 40 \mathrm{~L}$ to creatinine was higher than that of the WBC counts and the serum CRP levels. On the ROC curve, the sensitivity of the serum ratio of CD40L to creatinine was equal to that of the serum CRP levels, and the ratio's specificity was superior to that of the serum CRP level. 

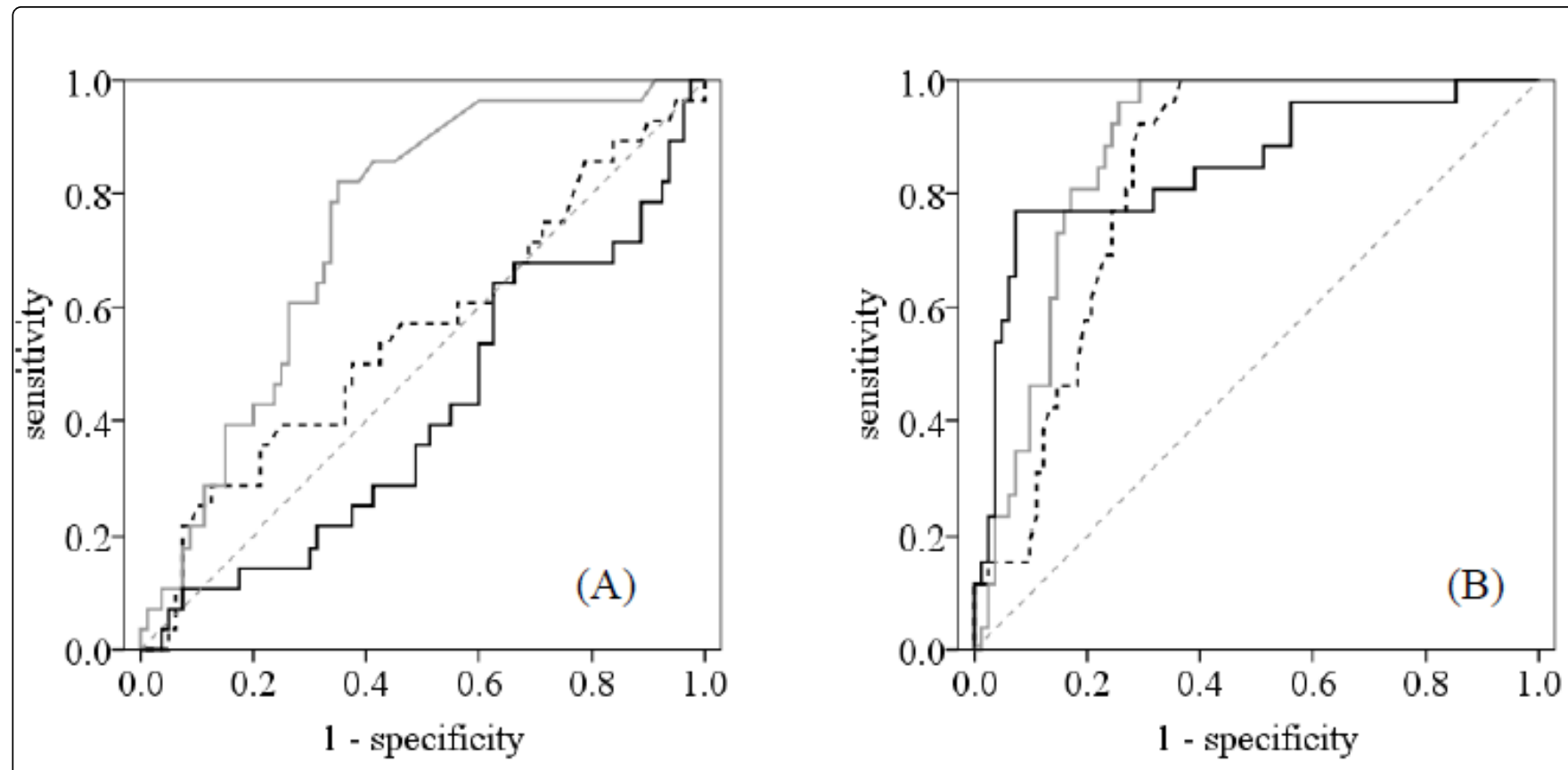

\begin{tabular}{|c|c|c|c|c|c|}
\hline & $\begin{array}{l}\text { Area under } \\
\text { the curve }\end{array}$ & $\begin{array}{c}95 \% \text { confidence } \\
\text { interval }\end{array}$ & $\begin{array}{c}\text { Optimum } \\
\text { cut-off levels }\end{array}$ & $\begin{array}{c}\text { Sensitivity } \\
(\%)\end{array}$ & $\begin{array}{c}\text { Specificity } \\
(\%)\end{array}$ \\
\hline \multicolumn{6}{|l|}{ (A) Disease activity } \\
\hline White blood cell counts & 0.555 & $0.425-0.685$ & $8850 / \mathrm{mm}^{3}$ & 57.1 & 54.7 \\
\hline Serum CRP levels & 0.730 & $0.634-0.826$ & $0.49 \mathrm{mg} / \mathrm{dL}$ & 85.7 & 61.6 \\
\hline Serum ratio of $\mathrm{sCD} 40 \mathrm{~L} /$ creatinine & 0.355 & $0.232-0 . \overline{478}$ & $13.6 \mathrm{ng} / \mathrm{mg}$ & 67.9 & 27.9 \\
\hline \multicolumn{6}{|l|}{ (B) Diagnosis of infection } \\
\hline White blood cell counts & 0.838 & $0.766-0.911$ & $9300 / \mathrm{mm}^{3}$ & 90.3. & 73.5 \\
\hline Serum CRP levels & 0.883 & $0.823-0.942$ & $1.36 \mathrm{mg} / \mathrm{dL}$ & 96.8. & 77.1 \\
\hline Serum ratio of $s C D 40 \mathrm{~L} /$ creatinine & 0.949 & $0.906-0.992$ & $99.7 \mathrm{ng} / \mathrm{mg}$ & 96.8 & 92.8 \\
\hline
\end{tabular}

Figure 3: The comparative ROC curves for three measurements for disease activity (A) and the diagnosis of infection (B). Solid black line $=$ ratio of serum-soluble CD40 ligand/creatinine. Solid gray line $=$ serum C-reactive protein levels. Dotted gray line $=$ WBC counts. Dotted black line $=$ the reference line.

\section{Discussion}

Although in the present study the serum-soluble CD40L levels in the inactive vasculitis patients with infectious complications were elevated and those levels were correlated with the serum CRP levels, the serum-soluble CD40L levels were also correlated with the serum creatinine levels. Serum-soluble CD40L levels may be associated with not only the degree of inflammation but also renal function. There have been only a few investigations of CD40L in patients with impaired renal function, and the high level of soluble CD40L in patients treated with hemodialysis was shown to result in a high risk of nonfatal/fatal atherothrombotic events [15]. Considering that the CD40/CD40L system is also expressed in cells of the vascular wall (endothelial cells, fibroblasts and smooth muscle cells) and an engagement of endothelial CD40 by CD40L induces a proinflammatory and thrombotic response [16], the elevated serum level of soluble CD40L in hemodialysis patients may be associated with an excessive production of the soluble form of CD40L from monocytes or platelets induced by inflammation.
On the other hand, the serum levels of low-molecular-weight proteins are known to correlate with serum creatinine levels. These proteins are filtrated at the glomerulus and reabsorbed and catabolized by proximal tubular cells, and the serum levels of such proteins in renal insufficiency patients are elevated due to low urinary filtration. Considering that the molecular weight of soluble CD40L is $15-18 \mathrm{kDa}$ [17], it is possible that the increase of serum-soluble CD40L levels in patients with renal insufficiency is caused by not only microinflammation but also low urinary excretion. However, the mechanisms underlying the elevated serum-soluble CD40L levels in patients with impaired renal function cannot be clarified at present, and further examination is needed.

Although CD40L was originally identified on stimulated $\mathrm{CD} 4^{+} \mathrm{T}$ cells and later on stimulated mast cells and basophils, platelets express CD40L within seconds of activation in vitro and in the process of thrombus formation in vivo [18]. In a large 2011 cohort study of GPA patients, several cytokines such as P-selectin, vascular endothelial growth factor, IL-8, and soluble CD40L were chosen as candidate biomarkers for measuring disease activity in GPA, because those 
cytokines arise from within the circulation are associated with platelet activation and inflammatory response [13]. In that cohort study, the strongest associations for active disease at baseline versus remission were observed for soluble CD40L. However, in the present study, the serum CD40L level did not reflect the disease activity of MPO-AAV. In that cohort study [13], the association of soluble CD40L was driven mainly by the association with the granulomatous but not the vasculitic components of BVAS/WG. Although soluble CD40L may reflect granulomatous formations in GPA (PR3-AAV), it is not apparently associated with vasculitis activity. Thus, there may be no elevation of soluble CD40L at the active phase of MPA (MPO-AAV), which is not accompanied by granulomatous formations.

In the present study, the soluble CD40L levels, in particular those levels corrected by serum creatinine, were elevated in the patients with infectious complications. Soluble CD40L levels were elevated during sepsis [19] and several infections such as meningococcal infection [20], pulmonary tuberculosis [21] and African tick bite fever [22]. In an abdominal sepsis model using cecal ligation and puncture (CLP), the plasma levels of soluble CD40L increased and the surface expression of CD40L on platelets decreased [23]. In platelet-depleting antibodytreated CLP mice, the CD40L levels were reduced by $90 \%$. Bacterial antigen-stimulated platelets in platelet-rich plasma released soluble CD40L, and bacterial antigen-stimulated peripheral blood mononuclear cells also released soluble CD40L [22]. Those studies suggested that elevated serum-soluble CD40L in sepsis was shown to result in the platelet shedding of CD40L. However, in the present study, there was no correlation between the soluble CD40L levels and the platelet counts.

On the other hand, the in vivo administration of lipopolysaccharide led to an activation of platelets and monocytes with the upregulation of CD40L on platelets, but soluble CD40L levels did not change significantly [24]. Moreover, it was recently demonstrated that neutrophil-derived matrix metalloproteinase- 9 controlled by RASrelated $\mathrm{C} 3$ botulinus toxin substrate 1 (Rac1) was an important regulator of CD40L shedding from platelets in sepsis [25]. Considering those studies, the elevated serum-soluble CD40L levels in sepsis may not depend on platelet counts or the degree of expression on platelets, and the levels may be related to the molecules cleaving CD40L. We could not determine how CD40L was released by platelets, monocytes or other cells in our present investigation. Further studies are needed to clarify the mechanisms underlying elevated soluble CD40L in patients with infectious complications.

Although the difference between the present active-vasculitis patients and the inactive-vasculitis patients without infectious complications was not significant, elevated serum levels of soluble CD40L and elevated ratios of serum-soluble CD40L to creatinine were observed in several patients with active vasculitis (Figures 1 and 2). Patients with active vasculitis may have an overlapping infection before and/or at onset, although patients with active vasculitis are usually treated with antibiotics therapy before the initiation of immunosuppressive therapy. In the present study, some of the activevasculitis patients who had elevated levels of serum-soluble CD40L may still have been complicated with infectious diseases. In the abovementioned cohort study [13], the serum soluble CD40L levels in PR3AAV patients were higher than those at the remission phase, but the serum soluble CD40L level before a disease flare-up was higher than that during the flare-up. We therefore speculated that granulomatous formation or bacterial infection may be associated with the elevation of CD40L in AAV.
To detect the presence of infectious complications regardless of vasculitis activity, several biomarkers such as procalcitonin [26] and serum high mobility group box 1 (HMGB1) [27,28] have been investigated. However, procalcitonin was elevated in several active AAV cases without infection [26], and serum HMGB1 levels were significantly higher in GPA patients [27] and were reported to correlate with the volume of pulmonary granuloma [28]. We previously demonstrated the efficacy of serum-soluble TREM-1 to detect the presence of infectious complications in AAV patients [29], but it depended on the patients' renal function, similar to CD40L. Therefore, more useful biomarkers are needed to detect the presence of infectious complications regardless of disease activity in AAV.

Our study has several limitations. First, the study population was limited to patients with MPO-ANCA-associated renal vasculitis. Further studies are necessary to compare patients with PR3-AAV or non-renal vasculitis. Moreover, all of the patients were Japanese, and the potential for genetic bias may prevent the applicability of our results to non-Japanese populations. In addition, it is necessary to confirm that the calculation of soluble CD40L divided by serum creatinine is suitable, which would require a larger number of control (without vasculitis or infection) with varying levels of renal function. Finally, this study was a retrospective cross-sectional study; a larger prospective longitudinal study (including vasculitis patients with relapse) would provide more definitive results.

In conclusion, we found no relationship between serum-soluble CD40L and disease activities or autoantibody production in MPOAAV. The serum levels of soluble CD40L may depend on both the patient's renal function and the existence of infectious complications, and this level may be a useful marker for the detection of infectious complications in MPO-AAV.

\section{Acknowledgement}

This study was supported in part by a Grant-in-Aid for Progressive Renal Diseases Research, Research on Intractable Disease, from the Ministry of Health, Labour and Welfare of Japan.

\section{Conflict of Interest}

The authors declare that they have no conflicts of interest.

\section{References}

1. Falk RJ, Terrell RS, Charles LA, Jennette JC (1990) Anti-neutrophil cytoplasmic autoantibodies induce neutrophils to degranulate and produce oxygen radicals in vitro. Proc Natl Acad Sci USA 87: 4115-4119.

2. Xiao H, Heeringa P, Hu P, Liu Z, Zhao M, et al. (2002) Antineutrophil cytoplasmic autoantibodies specific for myeloperoxidase cause glomerulonephritis and vasculitis in mice. J Clin Invest 110: 955-963.

3. Little MA, Smyth CL, Yadav R, Ambrose L, Cook HT, et al. (2005) Antineutrophil cytoplasm antibodies directed against myeloperoxidase augment leukocyte-microvascular interactions in vivo. Blood 106: 2050-2058.

4. Grewal IS, Flavell RA (1998) CD40 and CD154 in cell-mediated immunity. Annu Rev Immunol 16: 111-135.

5. Schönbeck U, Libby P (2001) The CD40/CD154 receptor/ligand dyad. Cell Mol Life Sci 58: 4-43.

6. Graf D, Müller S, Korthäuer U, van Kooten C, Weise C, et al. (1995) A soluble form of TRAP (CD40 ligand) is rapidly released after $\mathrm{T}$ cell activation. Eur J Immunol 25: 1749-1754. 
7. Kato K, Santana-Sahagún E, Rassenti LZ, Weisman MH, Tamura N, et al. (1999) The soluble CD40 ligand sCD154 in systemic lupus erythematosus. J Clin Invest 104: 947-955.

8. Goules A, Tzioufas AG, Manousakis MN, Kirou KA, Crow MK, et al. (2006) Elevated levels of soluble CD40 ligand (sCD40L) in serum of patients with systemic autoimmune diseases. J Autoimmun 26: 165-171.

9. Tamura N, Kobayashi S, Kato K, Bando H, Haruta K, et al. (2001) Soluble CD154 in rheumatoid arthritis: elevated plasma levels in cases with vasculitis. J Rheumatol 28: 2583-2590.

10. Jinnin M, Ihn H, Yazawa N, Asano Y, Yamane K, et al. (2003) Elevated circulating soluble CD40 ligand in patients with mixed connective tissue disease. Clin Rheumatol 22: 37-39.

11. Komura K, Sato S, Hasegawa M, Fujimoto M, Takehara K (2004) Elevated circulating CD40L concentrations in patients with systemic sclerosis. J Rheumatol 31: 514-519.

12. Wang CL, Wu YT, Liu CA, Lin MW, Lee CJ, et al. (2003) Expression of CD40 ligand on CD4+ T-cells and platelets correlated to the coronary artery lesion and disease progress in Kawasaki disease. Pediatrics 111: E140-147.

13. Tomasson G, Lavalley M, Tanriverdi K, Finkielman JD, Davis JC Jr, et al. (2011) Relationship between markers of platelet activation and inflammation with disease activity in Wegener's granulomatosis. J Rheumatol 38: 1048-1054.

14. Watts R, Lane S, Hanslik T, Hauser T, Hellmich B, et al. (2007) Development and validation of a consensus methodology for the classification of the ANCA-associated vasculitides and polyarteritis nodosa for epidemiological studies. Ann Rheum Dis 66: 222-227.

15. Hocher B, Liefeldt L, Quaschning T, Kalk P, Ziebig R, et al. (2007) Soluble CD154 is a unique predictor of nonfatal and fatal atherothrombotic events in patients who have end-stage renal disease and are on hemodialysis. J Am Soc Nephrol 18: 1323-1330.

16. Mach F, Schönbeck U, Sukhova GK, Bourcier T, Bonnefoy JY, et al. (1997) Functional CD40 ligand is expressed on human vascular endothelial cells, smooth muscle cells, and macrophages: implications for CD40-CD40 ligand signaling in atherosclerosis. Proc Natl Acad Sci U S A 94: 1931-1936.

17. Hsu YM, Lucci J, Su L, Ehrenfels B, Garber E, et al. (1997) Heteromultimeric complexes of CD40 ligand are present on the cell surface of human T lymphocytes. J Biol Chem 272: 911-915.

18. Henn V, Slupsky JR, Gräfe M, Anagnostopoulos I, Förster R, et al. (1998) CD40 ligand on activated platelets triggers an inflammatory reaction of endothelial cells. Nature 391: 591-594.
19. Lorente L, Martín MM, Varo N, Borreguero-León JM, Solé-Violán J, et al. (2011) Association between serum soluble CD40 ligand levels and mortality in patients with severe sepsis. Crit Care 15: R97.

20. Inwald DP, Faust SN, Lister P, Peters MJ, Levin M, et al. (2006) Platelet and soluble CD40L in meningococcal sepsis. Intensive Care Med 32: 1432-1437.

21. Mizusawa M, Kawamura M, Takamori M, Kashiyama T, Fujita A, et al. (2008) Increased synthesis of anti-tuberculous glycolipid immunoglobulin $\mathrm{G}(\mathrm{IgG})$ and IgA with cavity formation in patients with pulmonary tuberculosis. Clin Vaccine Immunol 15: 544-548.

22. Damås JK, Jensenius M, Ueland T, Otterdal K, Yndestad A, et al. (2006) Increased levels of soluble CD40L in African tick bite fever: possible involvement of TLRs in the pathogenic interaction between Rickettsia africae, endothelial cells, and platelets. J Immunol 177: 2699-2706.

23. Rahman M, Zhang S, Chew M, Ersson A, Jeppsson B, et al. (2009) Platelet-derived CD40L (CD154) mediates neutrophil upregulation of Mac-1 and recruitment in septic lung injury. Ann Surg 250: 783-790.

24. Kälsch T, Elmas E, Nguyen XD, Suvajac N, Klüter H, et al. (2007) Endotoxin-induced effects on platelets and monocytes in an in vivo model of inflammation. Basic Res Cardiol 102: 460-466.

25. Hwaiz R, Rahman M, Zhang E, Thorlacius H (2014) Rac1 regulates platelet shedding of $\mathrm{CD} 40 \mathrm{~L}$ in abdominal sepsis. Lab Invest 94: 1054-1063.

26. Moosig F, Csernok E, Reinhold-Keller E, Schmitt W, Gross WL (1998) Elevated procalcitonin levels in active Wegener's granulomatosis. J Rheumatol 25: 1531-1533.

27. Wibisono D, Csernok E, Lamprecht P, Holle JU, Gross WL, et al. (2010) Serum HMGB1 levels are increased in active Wegener's granulomatosis and differentiate between active forms of ANCA-associated vasculitis. Ann Rheum Dis 69: 1888-1889.

28. Henes FO, Chen Y, Bley TA, Fabel M, Both M, et al. (2011) Correlation of serum level of high mobility group box 1 with the burden of granulomatous inflammation in granulomatosis with polyangiitis (Wegener's). Ann Rheum Dis 70: 1926-1929.

29. Hirayama K, Nagai M, Ebihara I, Shimohata H, Kobayashi M, et al. (2011) Serum ratio of soluble triggering receptor expressed on myeloid cells- 1 to creatinine is a useful marker of infectious complications in myeloperoxidase-antineutrophil cytoplasmic antibody-associated renal vasculitis. Nephrol Dial Transplant 26: 868-874. 